Meta

Journal des traducteurs

Translators' Journal

\title{
Planning, Programming et Scheduling
}

\section{Lisette Ménard-Lépine}

Volume 25, numéro 3, septembre 1980

URI : https://id.erudit.org/iderudit/003467ar

DOI : https://doi.org/10.7202/003467ar

Aller au sommaire du numéro

Éditeur(s)

Les Presses de l'Université de Montréal

ISSN

0026-0452 (imprimé)

1492-1421 (numérique)

Découvrir la revue

Citer cet article

Ménard-Lépine, L. (1980). Planning, Programming et Scheduling. Meta, 25(3),

353-356. https://doi.org/10.7202/003467ar d'utilisation que vous pouvez consulter en ligne.

https://apropos.erudit.org/fr/usagers/politique-dutilisation/ 


\section{PLANNING, PROGRAMMING ET SCHEDULING}

La consultation de différents auteurs nous amène à constater plusieurs recoupements, dans le domaine de la gestion, entre les notions de PLANNING, PROGRAMMING et SCHEDULING, ainsi qu'une certaine confusion dans l'emploi des termes français correspondants. Nous étudierons chacune des notions pour en déterminer les équivalents.

\section{PLANNING}

Les auteurs anglais distinguent entre «long-range planning» et «short-range planning», PLANNING étant défini comme:

The act or process of making or carrying out plans; specif.: the establishment of goals, policies, and procedures for a social or economic unit (Webster's Third New International Dictionary).

En français, deux termes sont employés en ce sens: PLANIFICATION et PLANNING. En effet, dans l'entreprise, la PLANIFICATION ou le PLANNING désignent une fonction qui consiste à déterminer des objectifs précis et les moyens à mettre en ouvre pour les atteindre.

Le rôle de la planification est à la fois général et spécifique, à longue échéance et à court terme. Il s'agit de formuler, de déterminer et de mettre au point: les objectifs; les politiques; les plans; les programmes; les budgets; le calendrier des travaux; les normes; les méthodes; les procédures (sic) ; l'organisation; ainsi que les critères permettant de mesurer et d'évaluer les résultats et les contrôles requis pour obtenir les résultats voulus sans incohérences... (La Direction moderne, p. 23). Le processus complet de planning comporte plusieurs éléments qui peuvent se répartir en cinq grands groupes: les objectifs, les moyens pour atteindre les objectifs, les 
ressources requises, les systèmes de mise en cuvre, les mécanismes de contrôle (Le Management - Textes et cas, p. 126).

Nous avons donc deux termes qui, en français, agissent comme synonymes. Cependant, PLANNING, en plus de présenter l'inconvénient d'être emprunté à l'anglais, peut prêter à confusion car il sert aussi à désigner, dans un sens plus restreint:

Un programme détaillé et chiffré portant sur un élément quelconque de l'activité d'une entreprise (Le Petit Robert).

Ainsi, on rencontre des expressions telles que : planning financier, planning de fabrication, d'approvisionnement, d'embauche, d'étude du marché, etc., où le terme français PLANNING équivaut à l'anglais SCHEDULE.

De plus, il faut savoir distinguer entre «planification à long terme» et "planification à court terme», cette dernière pouvant correspondre à la programmation.

La planification à long terme est un effort dynamique entrepris pour déterminer et prévoir les principaux événements qui peuvent influer sur le sort de l'organisation. (...) La planification à court terme porte sur les activités spécifiques (programmes, calendriers, budgets, etc.) qui permettront d'obtenir dans l'avenir immédiat des résultats conformes aux objectifs à long terme (La Direction moderne, p. 36).

PROGRAMMING

Les dictionnaires généraux donnent de PROGRAMMING la définition suivante :

The planning, scheduling or performing of a program(Webster's Third New International Dictionary).

Et ils définissent PROGRAM comme étant :

A plan of procedure: a schedule or system under which action may be taken toward a desired goal: a proposed project or scheme (Ibid.).

En français, PROGRAMMATION s'entend de l'analyse et de l'établissement d'un programme.

Organisation systématique des actions à entreprendre ou des étapes à franchir pour atteindre effectivement des objectifs déterminés (Dictionnaire des sciences de la gestion, p. 264).

Le programme d'un projet (d'ingénierie) est l'ensemble des instructions précises dont l'exécution en séquence aux périodes prescrites avec des données appropriées fournit les informations nécessaires à la réalisation de l'ouvrage dans le délai imparti (L'Ingénierie et son organisation, p. 97).

À la lumière de ce qui précède, PROGRAMMING et PROGRAMMATION sont des équivalents stricts. Toutefois, il y a chevauchement entre les notions de PROGRAMMING et de SCHEDULING, en raison de la dimension temporelle, si bien que le terme PROGRAMMATION est souvent rendu par SCHEDULING comme, par exemple, dans l'expression «production scheduling» qui traduit «programmation de la production». 


\section{SCHEDULING}

En anglais, SCHEDULE peut désigner:

A usually written plan or proposal for future procedure typically indicating the objective proposed, the time and sequence of each operation, and the materials required (Webster's Third New International Dictionary).

Selon cette définition, SCHEDULE équivaut à PROGRAMME et l'opéra- , tion de SCHEDULING peut, par conséquent, être rendue par PROGRAMMATION.

Par ailleurs, on trouve dans le Handbook of Modern Office Management and Administrative Services une définition du «short-interval scheduling» qui précise davantage la notion:

A method for assigning a planned quantity of work to be completed by a specific time and is a means to determine that the quantity of work has been completed within the specified time limit (chap. 5, p. 111).

D'après cet usage, le SCHEDULING pourrait être assimilé à l'ORDONNANCEMENT, qui a pour but de prévoir et d'ordonner les conditions d'exécution d'un programme.

Pour prévoir les délais et obtenir leur respect, l'ordonnancement contrôle les charges de travail des différents services, relance ceux-ci en cas de retard et met en évidence toute anomalie dans l'avancement du travail pour permettre d'y remédier (Grand Larousse encyclopédique).

Toutefois, SCHEDULE peut aussi signifier «timetable»; l'expression SCHEDULING employée dans ce sens désigne alors: la fixation des délais d'exécution ou l'établissement du calendrier (des travaux).

\section{CONCLUSION}

La difficulté de traduction des termes étudiés tient au fait que, comme nous avons tenté de le démontrer :

- les termes anglais PROGRAMMING et SCHEDULING peuvent être traités comme des synonymes dans certains cas;

- le terme PLANNING utilisé en français peut avoir le sens de PLANIFICATION, mais il peut aussi signifier PROGRAMME;

- il faut savoir distinguer entre planification à long terme et planification à court terme;

- le chevauchement des notions de PLANIFICATION, PROGRAMMATION et ORDONNANCEMENT entraîne une certaine confusion.

En fait, les notions se chevauchent parce que dans les trois cas il est question d'objectifs et de délais. Cependant, on peut distinguer des étapes qui permettent de différencier les expressions. Il y a d'abord la PLANIFICATION, étape où l'on détermine, entre autres, des objectifs et un calendrier; pour que les objectifs déterminés soient atteints dans les délais fixés, on établit ensuite la PROGRAMMATION des différentes activités qui devront prendre 
place; et l'ORDONNANCEMENT permettra de faire respecter la programmation selon le calendrier établi.

TABLEAU RÉCAPITULATIF

\begin{tabular}{ll} 
ANGLAIS & FRANÇAIS \\
\hline planning & $\begin{array}{l}\text { planification } \\
\text { planning (sens large) }\end{array}$ \\
\hline programming & $\begin{array}{l}\text { programmation } \\
\text { planning (sens restreint) }\end{array}$ \\
\hline scheduling (schedule $=$ program) & $\begin{array}{l}\text { programmation } \\
\text { planning (sens restreint) }\end{array}$ \\
\hline $\begin{array}{l}\text { scheduling (schedule }=\text { timetable*) } \\
\text { calendar scheduling }\end{array}$ & $\begin{array}{l}\text { fixation des délais } \\
\text { établissement du calendrier }\end{array}$ \\
\hline scheduling (production control) & $\begin{array}{l}\text { ordonnancement } \\
\text { planning (sens restreint) }\end{array}$ \\
\hline
\end{tabular}

* Schedule presented in dated or undated format (in terms of working days numbers, for example).

LISETTE MÉNARD-LÉPINE

\section{BIBLIOGRAPHIE}

BONNY, H. B. and J. P. FREIN, Handbook of Construction Management and Organization, New York, Van Nostrand Reinhold, 1973.

CARLSON, D., La Direction moderne. Principes et méthodes, Paris, OCDE, 1963.

DUBUC, R., Vocabulaire de gestion, Montréal, Leméac, 1974.

Grand Larousse encyclopédique, Vol. 7, 1963.

HEYEL, V., Handbook of Modern Office Management and Administrative Services, McGraw-Hill, 1972.

LAURIN, P. et al., Le Management. Textes et cas, Montréal, McGraw-Hill, 1973.

LISSARAGUE, J., Qu'est-ce que le P.E.R.T.? Paris, Dunod, 1975.

MAYNARD, H. B., Handbook of Business Administration, McGraw-Hill, 1967.

MILLER, R. et al., L'Ingénieur, $\mathrm{N}^{\circ} 323$, janvier-février 1978, Montréal.

NASSER, F., L'Ingénierie et son organisation, Paris, Eyrolles, 1974.

ROMEUF, J. et J.-P. GUINOT, Manuel du chef d'entreprise, Paris, P.U.F., 1960

TEZENAS du MONTCEL, H., Dictionnaire des sciences de la gestion, Paris, Mame, 1972.

TEZENAS, J., Dictionnaire de l'organisation et de la gestion, Paris, Les Éditions d'Organisation, 1971.

The Random House Dictionary of the English Language, 1973.

Webster's New Collegiate Dictionary, 1975.

Webster's Third New International Dictionary, 1971. 